\title{
Effect of Dehydration on Dielectric Measurements of Biological Tissue as Function of Time
}

Gertjan Maenhout, Student Member, IEEE, Adam Santorelli, Member, IEEE, Emily Porter, Member, IEEE, Ilja Ocket, Member, IEEE, Tomislav Markovic, Member, IEEE, and Bart Nauwelaers Senior Member, IEEE

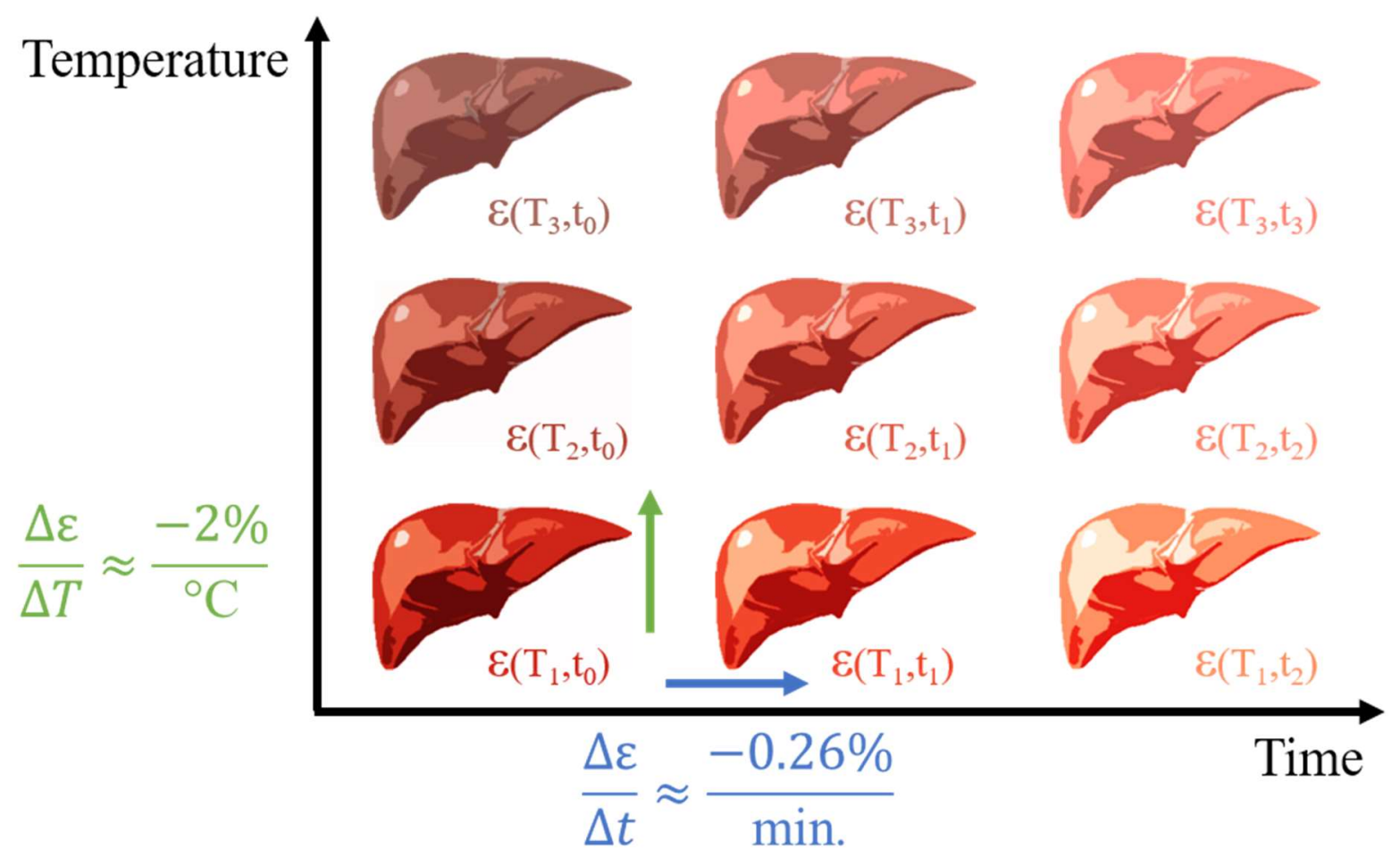

A graphical representation of the variability of the permittivity of the material under test depending on the environmental characteristics. Lazebnik et al. [10] have shown that a difference of $1^{\circ} \mathrm{C}$ can result in a change in dielectric properties up to $2 \%$. In this work, a change up to $9 \%$ in relative properties following an exponential decaying model is measured during a measurement interval of 35 minutes.

\section{Take-Home Messages}

- This work quantifies the influence of dehydration on dielectric properties and proposes a dehydrationmitigating technique to establish a more stable environment for longer measurement protocols.

- Due to the effect of dehydration, a relative change up to $9 \%$ in dielectric properties was established in a relevant temperature range during a relevant measurement period of 35 minutes.

- More precautions should be taken for dielectric measurement of biological tissue with regards to environmental conditions and other relevant metadata.

- For several applications relying on the difference in dielectric properties between healthy and malignant tissue, this error of $9 \%$ in measured data is on the same order of magnitude as the effects of interest. Thus, the effect of dehydration could severely obscure the expected difference in dielectric contrast.

- Until an agreement on a standard operating procedure with an acknowledged metadata form, we can only encourage authors to report all metadata in their work to allow future comparisons between studies of dielectric properties of biological tissue. 


\title{
Effect of Dehydration on Dielectric Measurements of Biological Tissue as Function of Time
}

\author{
Gertjan Maenhout, Student Member, IEEE, Adam Santorelli, Member, IEEE, Emily Porter, Member, IEEE, \\ Ilja Ocket, Member, IEEE, Tomislav Markovic, Member, IEEE, and Bart Nauwelaers, Senior Member, IEEE
}

\begin{abstract}
The lack of a standardized operating protocol for dielectric measurements of biological tissues makes it difficult to objectively compare reported dielectric properties since these properties are affected by multiple environmental confounders. This work investigates the effect of dehydration on dielectric properties as a function of time at different temperatures. Additionally, it proposes a dehydration mitigating technique. The dielectric properties of porcine liver samples were measured during a 35 minute measurement interval at different temperatures. A first set of six experiments was conducted in standard laboratory conditions. A second set of two experiments was conducted with a modified measurement setup with reduced air flow. Under standard conditions, relative changes in the dielectric properties up to $9 \%$ were observed. With the modified setup, the relative change was halved compared to standard conditions. This reduction indicates that the environmental conditions have a considerable influence and that modifying these conditions extends the stability of the dielectric properties over time. For several applications relying on the difference in dielectric properties between healthy and malignant tissue, an error of $9 \%$ in measured data is on the same order as the effects of interest. The expected difference in dielectric contrast could be obscured due to dehydration.
\end{abstract}

Keywords-dielectric measurements, nutrition and hydration, temperature dependence, biological cells, biological system modeling, quality management.

\section{INTRODUCTION}

$\mathbf{S}$ INCE the 1980's and before, electromagnetic techniques have been used for biomedical applications [1] and during recent years, more and more new applications have emerged. All these applications rely on the dielectric properties of the tissues: relative permittivity $\left(\epsilon_{r}\right)$ and the conductivity $(\sigma)$. Based on the differences in $\epsilon_{r}$ and $\sigma$, tissues can be classified and malignant or cancerous zones can be detected using different techniques, e.g. electrical impedance tomography [2] or more recently microwave imaging [3], [4]. Moreover, building on the well-known microwave heating capabilities, microwave hyperthermia [5] and microwave ablation applicators [6] are designed and their designs can be improved with more accurate knowledge of the dielectric properties of the tissues. The better these dielectric properties are known, the more efficient the malignant zone can be heated to the correct temperature without affecting the surrounding healthy tissue.

A first comprehensive overview of dielectric data for different tissues in a wide frequency band was given in 1996 [7]. It assessed the current state of dielectric data and exposed the gaps in the available data. Nowadays, the IT'IS database [8] is considered to be the largest database for dielectric data. Nevertheless, a standardized operating procedure (SOP) is lacking, which makes it hard to compare data from different experiments. In 2017, a first framework (MINDER) was introduced to include the minimum information for dielectric measurements of biological tissue [9]. A list of possible

G. Maenhout, I. Ocket, T. Markovic and B. Nauwelaers are with Division Telemic, Department of Electrical Engineering, KU Leuven, Kasteelpark Arenberg 10, Box 2444, 3001 Leuven, Belgium.

A. Santorelli and E. Porter are with Translational Medical Device Lab, $2^{\text {nd }}$ Floor Lambe Translational Research, National University of Ireland, Galway, Ireland.

I. Ocket and T. Markovic are also with imec, Kapeldreef 75, 3001 Heverlee, Belgium. confounders that can influence measurements, were described and summarized within this MINDER framework.

Two confounders are known to have a major impact on dielectric measurements. Firstly, it has been proven that the influence of temperature is significant and cannot be neglected: a difference of $1^{\circ} \mathrm{C}$ can possibly lead to a $2 \%$ change in dielectric properties [10]. A second confounder is the time between the excision of the sample and the time of the measurement. This includes among others the time for sample transportation, preparation, pre-heating and the actual measurement time. The combination of these two confounders, temperature and time, causes dehydration of the sample under test.

Apart from contributing to the standardization process, we must also consider the difference between in vivo dielectric properties versus ex vivo extracted dielectric properties. Since most of the aforementioned biomedical applications are minimally invasive procedures, they operate on in vivo tissue. Therefore, they require the dielectric properties from in vivo measurements for an optimal design and to fully test their capabilities. However, most measurements are executed on excised tissue samples and sometimes only ex vivo data is available. This raises the question how to minimize the difference between in vivo dielectric properties versus ex vivo extracted dielectric properties. If the sample is handled properly, the difference between in and ex vivo is not significant [11]. However, if the time between the excision and the measurement of the sample is too long such that the sample temperature drops to the ambient room temperature $\left(\sim 22^{\circ} \mathrm{C}\right)$, an in verus ex vivo difference of up to $30 \%$ could be observed given the reported temperature coefficients up to $\frac{\Delta \epsilon}{\epsilon}=\frac{2 \%}{{ }^{\circ} \mathrm{C}}$ [10], [12], [13]. If the temperature of the sample would drop after excision due to practicalities, e.g. transport, it might be worth considering to reheat it to a physiological temperature to have a closer match with the actual in vivo dielectric properties. However, the extra time 
for this reheating as well as the higher temperature contribute both to an increased dehydration rate. Therefore, the influence of this increased dehydration rate should be analyzed to better understand the effect of the environmental conditions on the extracted dielectric properties.

In this work, we investigate the influence of dehydration on the dielectric properties as a function of time at different temperatures and we propose a straightforward solution to limit this effect for future standardized data collection. Eight separate experiments were conducted. Each experiment monitored the relative change in dielectric properties due to dehydration of the tissue under test during an experimental period of 35 minutes. To investigate the dehydration rates as a function of temperature, experiments were conducted at different temperatures. A first set of six experiments at six different temperatures $\left(22.6^{\circ} \mathrm{C}-36.1^{\circ} \mathrm{C}\right)$ was conducted under standard conditions. A second set of two experiments $\left(35.2^{\circ} \mathrm{C}\right.$, $37.7^{\circ} \mathrm{C}$ ), was conducted with a modified measurement setup in which the air flow around the sample is limited by simply sealing the sample container. Both sets of experiments are described in detail in section II. In addition. Section III presents the measured data of all eight experiments. Section IV discusses the data as well as the observed differences and emphasizes the need for a standardized framework for the measurements of biological tissues.

\section{MATERIALS AND MEthods}

\section{A. Theory of Tissue Dehydration}

In order to characterize this dehydration process, a first approximation can be found in the food process engineering branch where the moisture content of the sample $X(t)$ is described over time by an exponential decay from an initial state $X_{\text {init. }}$ to an equilibrium state $X_{\text {eq. }}$ [14]:

$$
\begin{gathered}
X(t)=X_{\text {eq. }}-\left(X_{\text {eq. }}-X_{\text {init. }}\right) \times \exp \left(-\frac{t}{t_{\mathrm{c}}}\right), \\
X_{\text {eq. }}=b_{1} \times \exp \left(\frac{b_{2}}{273+T}\right) \times\left(\frac{y}{100-y}\right)^{b_{3}}, \\
t_{\mathrm{c}}=c_{0} \times d^{c_{1}} \times u^{-c_{2}} \times T^{-c_{3}} \times y^{c_{4}},
\end{gathered}
$$

where $t_{\mathrm{c}}[\mathrm{h}]$ is the time constant, $d[\mathrm{~m}]$ the thickness or radius of the sample, $u\left[\frac{\mathrm{m}}{\mathrm{s}}\right]$ the air velocity around the sample, $T\left[{ }^{\circ} \mathrm{C}\right]$ the temperature, $y[\%]$ the relative humidity and the values $b_{i}$ and $c_{i}$ are strictly positive empirical constants, experimentally derived in literature [14]. It is known that the water content has a serious impact on the dielectric properties of high water content tissues, e.g. liver and muscles [15], [16], since we are mainly measuring in the $\gamma$ dispersion region [17]. Therefore, we expect similar trends in dielectric properties as the ones that are described for the moisture content $X(t)$. It is evident that the equilibrium state for tissues with high water content will be lower than the initial state. Therefore, we must examine $t_{\mathrm{c}}$ in more detail to monitor the rate at which this dehydration process occurs. A back-of-the-envelope calculation with estimated conditions $\left(d=0.01 \mathrm{~m}, u=0.5 \frac{\mathrm{m}}{\mathrm{s}}\right.$, $T=30^{\circ} \mathrm{C}$ and $y=60 \%$ ) results in $t_{\mathrm{c}}$ equal to 2.25 hours. This means that after half an hour, which is a reasonable time for sample transportation and preparation, $20 \%$ of the water content is lost. This is a considerable amount and should thus be noticed during experiments.

Two studies regarding tissue dehydration were recently published. A first one measured the dielectric properties of mouse liver over time at an ambient temperature of $22^{\circ} \mathrm{C}$ [18]. The liver was stored in a sealed container in between measurements to limit excessive dehydration. After 3.5 hours, a dielectric change of more than $25 \%$ was noted in the real and imaginary part of the dielectric properties. A second study dehydrated mouse livers using a loss-on-drying technique to multiple desired dehydration values [19]. After this dehydration step at a temperature of $40^{\circ} \mathrm{C}$, the sample was allowed to return to an ambient temperature of $25^{\circ} \mathrm{C}$. A similar monotonic decreasing trend in the real and imaginary part of the dielectric properties was observed. However, no study to date has quantified the impact of dehydration mitigating techniques.

\section{B. Measurement Setup}

Porcine liver samples were purchased at a local butcher where it had arrived the same day from the abattoir. Before measurements, the liver was vacuum packed and stored in a fridge at $4^{\circ} \mathrm{C}$. After system calibration, the liver was taken out of the fridge and a sample of approximately $2 \mathrm{~cm} \times$ $2 \mathrm{~cm} \times 2 \mathrm{~cm}$ was prepared. The rest of the liver was placed back in the fridge and used for following samples. The freshly-cut sample was preheated in a sample container to the desired temperature on an aluminum platform with an embedded temperature control unit. During the preheating step, the sample was wrapped in aluminum foil to prevent dehydration prior to the experiment. A type $\mathrm{T}$ thermocouple (relative accuracy of $\pm 0.5^{\circ} \mathrm{C}$ ) was inserted $\pm 2 \mathrm{~mm}$ in the sample from the upper side, related to the sensing depth of the dielectric probe [20]. When the temperature readout remained stable for 2 minutes (readout accuracy of $0.1^{\circ} \mathrm{C}$ ), the sample was unwrapped and dried with a paper towel to prevent the build-up of any excess moisture at the probe tip. A lifting platform was used to raise the heater and the sample to contact the dielectric probe. After every single experiment, the used sample was disposed. The overall setup is shown in Fig. 1. A Texas Instrument sensor (HDC1080-EVM) measured the relative humidity in the lab $( \pm 60 \%)$.

During the first set of experiments, the sample container was uncovered and air could flow freely around the sample. Since air flow $u$ influences the time constant $t_{\mathrm{c}}$ according to (3), a straightforward solution to increase the time constant is to limit the air flow around the sample. Therefore, a second set of experiments was performed in which a cover was used to seal the sample container and thus to limit the air flow around the sample. The cover has two minor holes that allow access of the dielectric probe and the thermocouple.

A Keysight slim form open-ended coaxial probe (N1501A) was used for the measurement of the dielectric properties. The probe was connected to a Keysight PNA (E8361A) using the included Keysight connector saver adapter and a $2.4 \mathrm{~mm}$ coaxial cable. The power level of the PNA was set to $-5 \mathrm{dBm}$ and an IF bandwidth of $30 \mathrm{~Hz}$ was used as recommended 


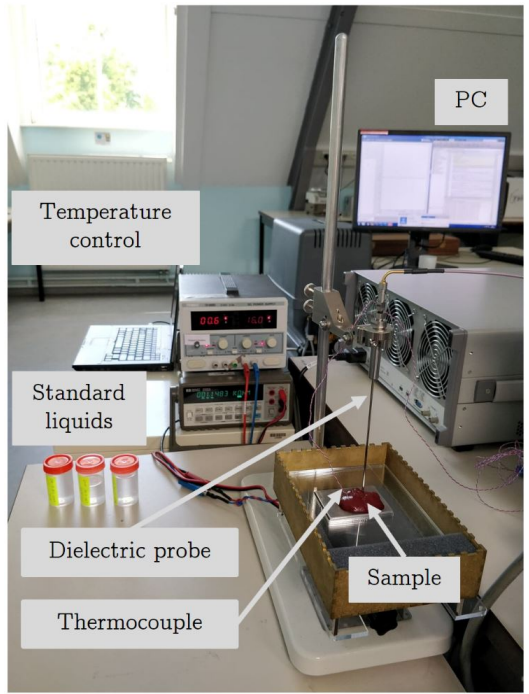

Fig. 1: Overview of the measurement setup with the temperature control unit for heating and the the thermoucouple and $\mathrm{PC}$ for sample temperature monitoring.

in the manual of the probe. Measurements were executed in a frequency range from $500 \mathrm{MHz}$ to $20 \mathrm{GHz}$ with a frequency step of $50 \mathrm{MHz}$. In every single experiment, the PNA performed 30 measurements with a one-minute timeinterval between subsequent measurements.

Throughout the 30 dielectric measurements in every single experiment, the probe remained continuously in contact with the sample to eliminate all possible measurement variance related to sample heterogeneity at different locations and to a different probe-to-sample contact pressure. However, since dehydration takes place at the surface of the sample, leaving the probe in direct contact will thus decrease the rate of dehydration at the most important sensing zone, i.e. the zone beneath the dielectric probe. Nevertheless, this option was preferred in order to ensure that the observed changes could only be attributed to the dehydration process itself. The measurement results will thus represent a lower dehydration rate than what can be expected during typical measurements where the probe is placed on and taken off the tissue multiple times throughout the experiment and where the complete surface area experiences an equal, and thus higher, dehydration rate than the one that is observed here.

\section{Experimental Analysis}

The dielectric probe was calibrated with the Keysight software using an open (leaving the probe in the air), short (connecting the provided calibration short) and a load (inserting the probe in deionized water at a known temperature). The probe tip was always immersed more than $5 \mathrm{~mm}$ in the calibration or validation liquids with respect to Keysight guidelines and the known sensing volume of the used probe [20]. Before and after every single experiment of approximately 30 minutes, pre- and post-validation measurements were performed on a $0.1 \mathrm{M} \mathrm{NaCl}$ solution for error analysis and uncertainty assessment [21]. This saline solution was chosen since it is the
TABLE I: Overview of the conditions of the conducted experiments with the corresponding mean $(\overline{\mathrm{T}})$ and standard deviation $\left(s_{\mathrm{T}}\right)$ of the temperature of the sample under test. A fresh sample was used in every single experiment.

\begin{tabular}{|c|c|c|c|}
\hline Exp. nb. & Air flow & $\overline{\mathrm{T}}\left[{ }^{\circ} \mathbf{C}\right]$ & $\boldsymbol{s}_{\mathrm{T}}\left[{ }^{\circ} \mathbf{C}\right]$ \\
\hline 1 & Standard (uncovered container) & 22.6 & 0.1 \\
\hline 2 & Standard (uncovered container) & 24.5 & 0.1 \\
\hline 3 & Standard (uncovered container) & 29.6 & 0.3 \\
\hline 4 & Standard (uncovered container) & 32.8 & 0.4 \\
\hline 5 & Standard (uncovered container) & 34.0 & 0.1 \\
\hline 6 & Standard (uncovered container) & 36.1 & 0.2 \\
\hline 7 & Reduced (sealed container) & 35.2 & 0.2 \\
\hline 8 & Reduced (sealed container) & 37.7 & 0.4 \\
\hline
\end{tabular}

commonly used validation liquid [22], [23]. Its temperature, over all validation measurements, was measured with a type $\mathrm{T}$ thermocouple $\left(21.5^{\circ} \mathrm{C}-22.6^{\circ} \mathrm{C}\right)$. Antiseptic pads were used to clean the probe between different measurements to avoid cross-contamination of the sample and the calibration and validation liquids.

The uncertainty at every frequency point was calculated as the combination of three equally contributing sources of error: the random uncertainty, the systematic offset compared to a known material and drift throughout the experiment. These uncertainties are all obtained by a statistical analysis of a series of observations [24], [25] and can thus be classified as type $\mathrm{A}$ uncertainties. The $0.1 \mathrm{M} \mathrm{NaCl}$ validation liquid was measured five times before and after every experiment. The random uncertainty was calculated as the mean of the standard deviation of the permittivity value of the pre- and the postvalidation measurements ( $s_{\text {pre. }}$ and $\left.s_{\text {post. }}\right)$. To estimate the systematic uncertainty, the mean permittivity value of the five measurements of the validation liquid before the experiment $\left(\overline{\epsilon_{\mathrm{val}}}\right)$ was compared to values found in literature $\left(\epsilon_{\text {lit. }}\right)$ [23] and, due to the scarce amount of information, a rectangular distribution was assumed in the interval $\left[\overline{\epsilon_{\mathrm{val}}}-\epsilon_{\text {lit. }}, 0\right]$ or $\left[0,\left(\overline{\epsilon_{\mathrm{val}}}-\epsilon_{\mathrm{lit} .}\right)\right]$, for respectively an underestimation or an overestimation [25]. At last, the drift during the experiment was assessed as the difference between the systematic error before $\left(\Delta_{\text {pre. }}=\left(\overline{\epsilon_{\text {val.pre. }}}-\epsilon_{\text {lit.pre. }}\right)\right)$ and the systematic error after the experiment $\left(\Delta_{\text {post. }}=\left(\overline{\epsilon_{\text {val.post. }}}-\epsilon_{\text {lit.post. }}\right)\right)$, also under the assumption of a rectangular distribution. The combined standard deviation $s_{\text {comb. }}$ is calculated as follows:

$$
\begin{aligned}
s_{\text {comb. }}^{2}=\left(\frac{s_{\text {pre. }}+s_{\text {post. }}}{2}\right)^{2} & +\left(\frac{\overline{\epsilon_{\text {val. }}}-\epsilon_{\text {lit. }}}{2 \times \sqrt{3}}\right)^{2} \\
& +\left(\frac{\Delta_{\text {pre. }}-\Delta_{\text {post. }}}{2 \times \sqrt{3}}\right)^{2} .
\end{aligned}
$$

Using a coverage factor of $k=2$, the actual permittivity value should be located within the proposed error bars with an expanded uncertainty.

\section{RESULTS}

In total, eight different experiments were performed on the aforementioned setup, each at a different temperature. During the first set of six experiments, the sample was exposed to the standard airflow in the laboratory while during the second set of two experiments, the sample container was sealed and the 

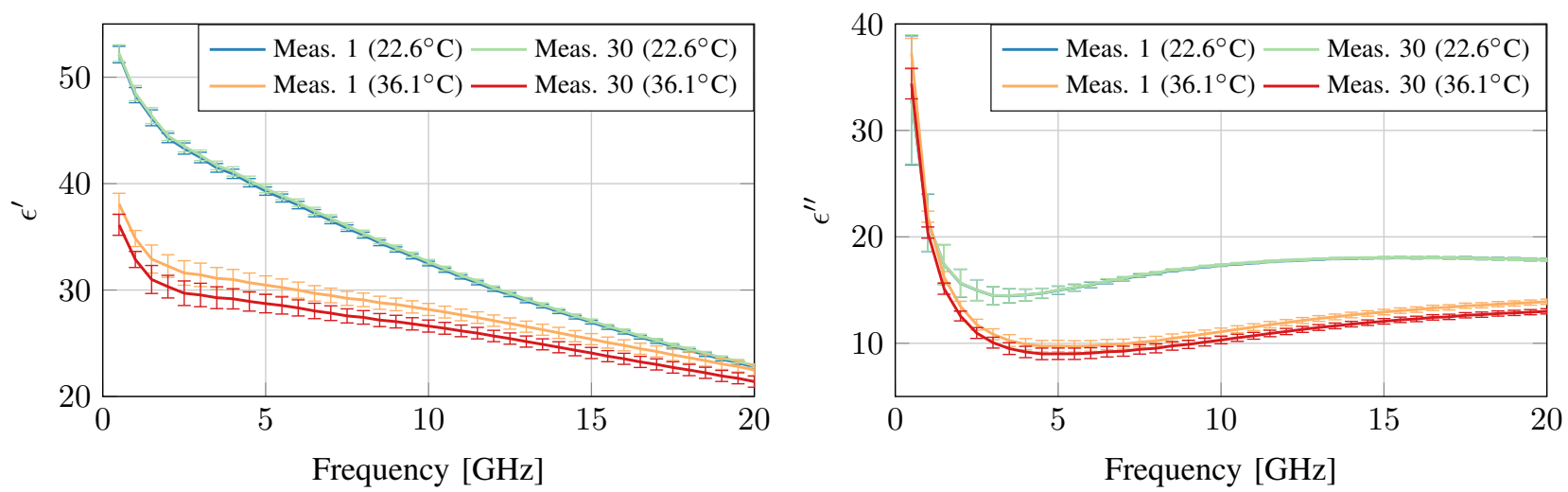

Fig. 2: The extracted complex permittivity $\left(\epsilon^{\prime}-\mathrm{j} \epsilon^{\prime \prime}\right)$ of the sample under test at dielectric measurement $1(0 \mathrm{~min}$.) and at dielectric measurement 30 (34.9 min.) of experiments number 1 and 6 , at $22.6^{\circ} \mathrm{C}$ and $36.1^{\circ} \mathrm{C}$ respectively, with their corresponding error bars obtained from the error assessment as explained in section II-C.

air flow around the sample was thus reduced. An overview of all conducted experiments is presented in Table I. In this section, the data of the experiment at the highest temperature and closest to the physiological temperature $\left(36.1^{\circ} \mathrm{C}\right)$ under standard conditions, experiment number 6 , is analyzed in detail and a compact overview of all experiments is presented.

The temperature of the sample was monitored throughout the complete experiment with a type $\mathrm{T}$ thermocouple. This was inserted in the sample as close as possible to the the contact point of the dielectric probe. The standard deviation of the temperature throughout experiment number 6 was $0.2^{\circ} \mathrm{C}$ and is maximum $0.4^{\circ} \mathrm{C}$ in all other experiments (Table I). Therefore, temperature changes could be responsible for a relative change in dielectric characteristics of $0.2 \%$ to $0.8 \%$ [10], [12], [13].

Throughout the measurement time, dielectric measurements are performed at thirty consecutive time points and their uncertainty is assessed as explained in section II-C. In Fig. 2, the complex dielectric permittivity $\left(\epsilon^{\prime}-\mathrm{j} \epsilon^{\prime \prime}\right)$ over the entire frequency range is extracted and presented with the corresponding error bars for the lowest and highest measured temperature $\left(22.6^{\circ} \mathrm{C}\right.$ and $\left.36.1^{\circ} \mathrm{C}\right)$. At $22.6^{\circ} \mathrm{C}$, the mean uncertainty value over the complete frequency range and its associated standard deviation is $0.81 \pm 0.24 \%$ and $2.28 \pm 3.45 \%$, respectively for the real and imaginary permittivity values. The error bars of the first and last measurement overlap. We thus cannot conclude that dehydration during 35 minutes causes a significant difference in dielectric properties at this temperature. At $36.1^{\circ} \mathrm{C}$, an uncertainty over the complete frequency range of $2.40 \pm 0.51 \%$ and $3.22 \pm 1.33 \%$ is obtained for respectively the extracted real and imaginary values. It is clear from Fig. 2 that the first and the last performed measurement are statistically significantly different due to their non-overlapping error bars.

In Fig. 3, the relative values of $\epsilon^{\prime}$ and $\epsilon^{\prime \prime}$, with respect to the first measurement, are presented with respect to time. At four linearly spaced frequencies throughout the measured frequency range, the relative value of the dielectric permittivity is displayed during the entire experiment duration of 35 minutes. As expected from (1), an exponential decay is observed at all frequencies for the real as well as the imaginary part of the dielectric properties. We observed a similar exponential decay at every frequency point throughout the complete frequency range. This corresponds to other observations in literature [19].

An overview of all obtained results is shown in Fig. 4. Since the dehydration process results in a similar decay over all frequencies, all conducted experiments are compared at a single frequency of $10 \mathrm{GHz}$, in the middle of the measured frequency range. A surface represents the relative $\epsilon^{\prime}$ and $\epsilon^{\prime \prime}$ values of the experiments with a standard airflow (experiments 1 to 6) at every temperature value throughout the entire duration of the experiments. On the other hand, the solid red lines represent the relative data obtained in the second set of experiments with a reduced airflow (experiments 7 to 8 ).

\section{Discussion AND Future Work}

Based on Fig. 4, three conclusions can be drawn. Firstly, a decrease of $\epsilon^{\prime}$ and $\epsilon^{\prime \prime}$ values is observed throughout time for each measured temperature value for both sets of experiments (uncovered and sealed). This means that the longer the time interval between sample excision and measurement, the more the dielectric properties of the sample under test will decrease. This variation follows an exponential decay as explained before. Secondly, we can observe that the variation over time is temperature dependent: the change of $\epsilon^{\prime}$ and $\epsilon^{\prime \prime}$ values at higher temperatures is much more pronounced than the one at lower temperatures as expected. According to (1)-(3), these changes should follow an exponential trend with respect to temperature. Therefore, an exponential decaying curve is fitted through the relative values of the first set of experiments at the end of each experiment (experiments 1 to 6). This curve is presented as the dashed red curve in Fig. 4. Thirdly, a difference is observed between the standard experiments and the ones with a reduced airflow. Note that the experiments with a reduced airflow were only conducted at higher temperatures since the largest, statistically significant relative changes were observed there during the set of standard experiments. In reference to the fitted exponential decaying curve, the expected relative $\epsilon^{\prime}$ and $\epsilon^{\prime \prime}$ values were calculated at the temperature values of the experiments performed with reduced air flow and are represented as red dots in Fig. 4. Based on this extrapolated 

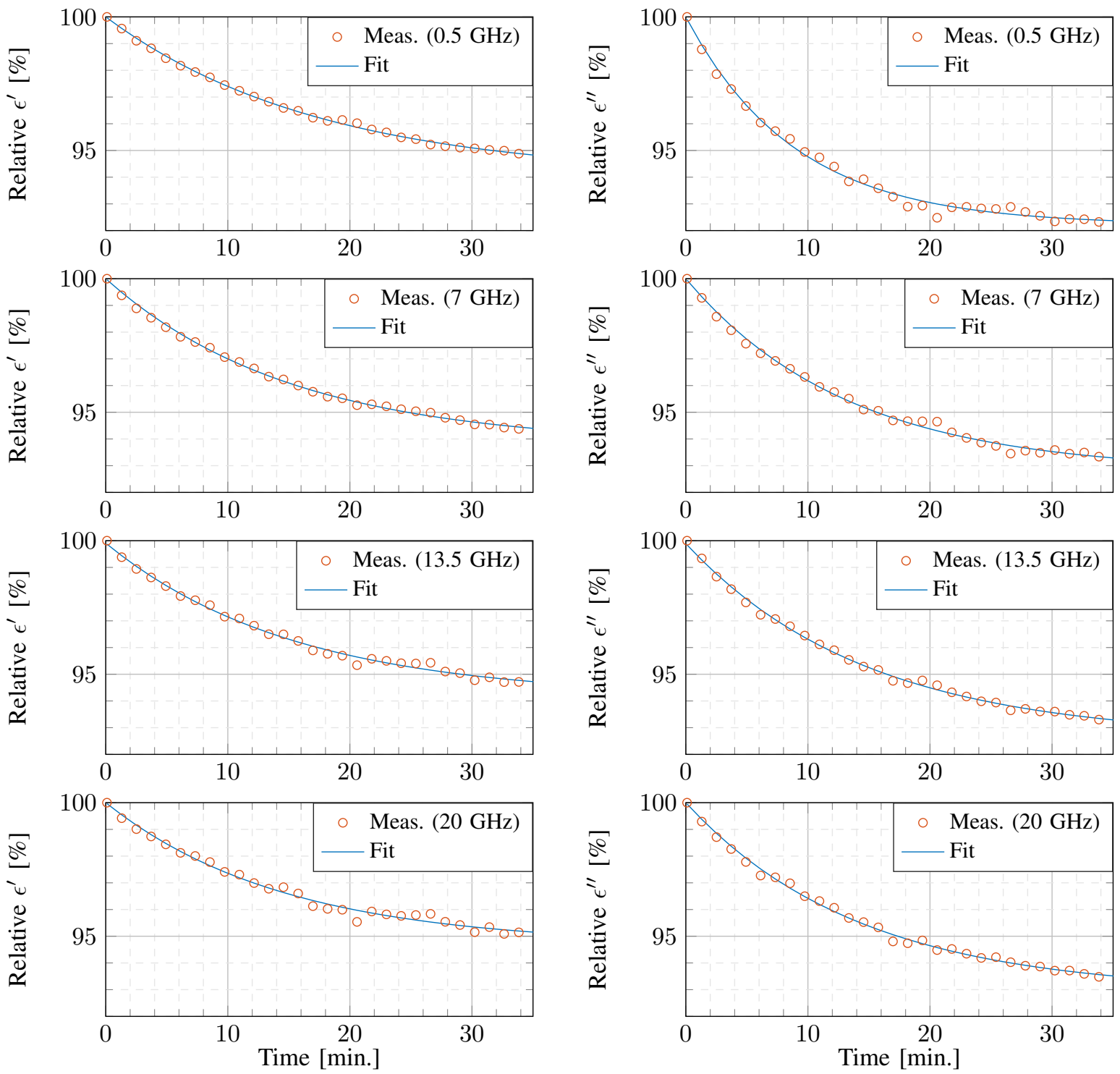

Fig. 3: The measured real $\left(\epsilon^{\prime}\right)$ and imaginary $\left(\epsilon^{\prime \prime}\right)$ part of the extracted complex permittivity throughout experiment number 6 (conducted at $36.1{ }^{\circ} \mathrm{C}$ ), evaluated at four linearly spaced frequencies, and fitted with an exponential decaying model.

curve, a relative change of $5.0 \%$ and $6.6 \%$ for $\epsilon^{\prime}$ and $6.4 \%$ and $8.4 \%$ for $\epsilon^{\prime \prime}$ was expected at $35.2^{\circ} \mathrm{C}$ and $37.7^{\circ} \mathrm{C}$, respectively. Nonetheless, the relative change of $\epsilon^{\prime}$ and $\epsilon^{\prime \prime}$ measured on the reduced air flow measurement setup only amounts to $1.9 \%$ and $2.1 \%$ in the case of $\epsilon^{\prime}$ and $1.7 \%$ and $4.1 \%$ in the case of $\epsilon^{\prime \prime}$, which is significantly lower than the expected values. This difference (3.1\% and $4.5 \%$ in the case of $\epsilon^{\prime}$ and $4.7 \%$ and $4.3 \%$ in the case of $\epsilon^{\prime \prime}$ ) is indicated with double sided yellow arrows in Fig. 4. Therefore we can conclude that storing the tissue samples in a sealed container throughout the measurements has thus a positive influence on the stability of the sample over time. However, this is unfortunately not yet embedded in all different dielectric tissue measurements protocols, which is once again an additional reason to pursue a standard operating protocol and a reporting policy for dielectric measurements of biological tissue.

This work demonstrated the effect of dehydration as a function of time on dielectric measurements of porcine liver at different temperatures. Due to the high water content present in these samples, our initial expectations were confirmed and we observed a similar behavior in dielectric properties as the one predicted for the moisture content $X$ with respect to time $t$, temperature $T$ and air flow $u$. The aforementioned trends concerning $t, T$ and $u$ suggest that equations from food process engineering can be used to predict the behavior of dielectric properties and assess the possible influence of other confounders. Furthermore, under the assumption that freshly excised tissue samples are subject to the same physical principle of dehydration as a function of time, the use of these equations might open up possibilities to predict the in vivo dielectric properties from multiple ex vivo dielectric measurements over time with appropriate fitting models.

As mentioned before, a lower dehydration rate is expected in this work compared to standard measurements due to the 


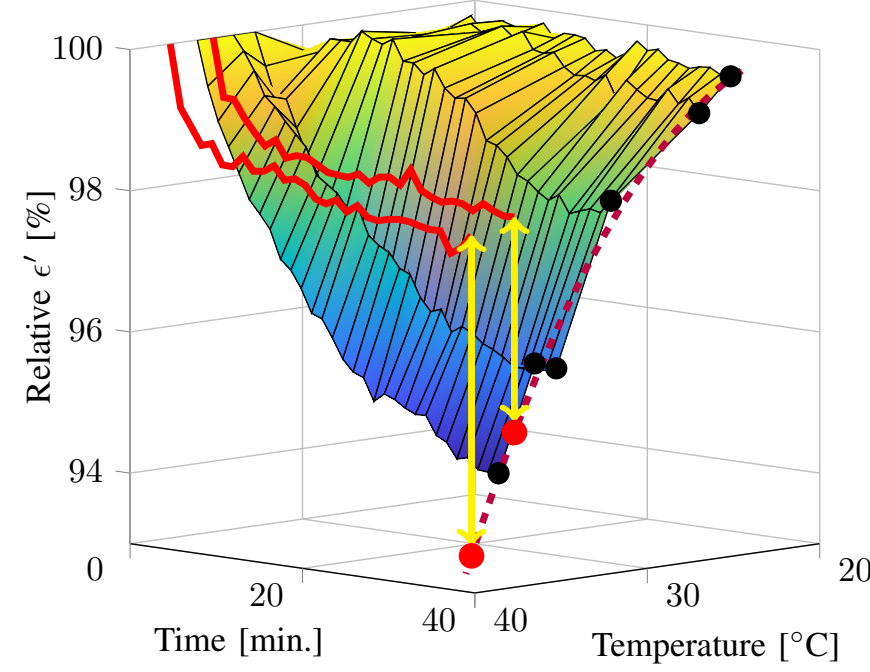

(a)

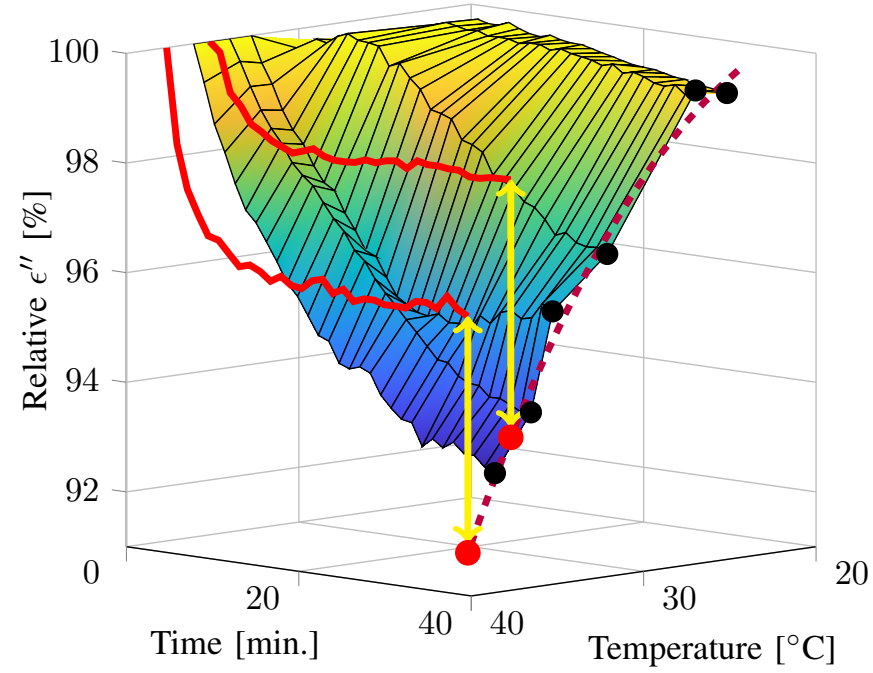

(b)

Fig. 4: The surface represents the relative variation in $\epsilon^{\prime}$ and $\epsilon^{\prime \prime}$ of all conducted experiments in standard conditions (experiments 1 to 6 ) over time at a single frequency of $10 \mathrm{GHz}$. A dashed dark red line represents the exponential fit through the relative $\epsilon^{\prime}$ and $\epsilon^{\prime \prime}$ values at the end of each of these experiments (black dots). The solid red lines represent the data from the experiments with a reduced air flow (experiments 7 to 8). The double sided yellow arrows indicate the difference at the end of each experiment with reduced airflow between the measured relative $\epsilon^{\prime}$ and $\epsilon^{\prime \prime}$ values with respect to the extracted values (red dots) from the exponential fit at the corresponding temperatures.

continuous contact of the probe. Nevertheless, a significant change in relative $\epsilon^{\prime}$ and $\epsilon^{\prime \prime}$ values up to $9 \%$ is observed during a limited measurement period of 35 minutes at a physiological temperature. Therefore, if ex vivo obtained dielectric properties are used (due to the lack of in vivo available data) for designing minimally invasive in vivo applications or therapeutical procedures that rely on the difference in dielectric properties between healthy and malignant tissue, this possible error of up to $9 \%$ (if not taken into consideration) is on the same order of magnitude as the effects of interest. For example, the reported difference between healthy and malignant breast tissue is approximately $10 \%$ or less [26], [27] and a difference of roughly 5\% was reported for healthy and malignant colon tissue [28]. If samples of healthy and malignant tissue were measured at different times from excision, this could obscure the expected difference in dielectric contrast between them and thus influence all derived applications. Since the effect of dehydration is even visible at room temperature as demonstrated in literature [18], we advise to reduce the time interval between sample excision and the measurement to a minimum as well as to store the sample in a sealed container to limit the air flow around the sample. If future dielectric measurements would be performed at body temperature [29] to close the gap between ex vivo measurements and in vivo properties caused by a difference in temperature, then the effect of dehydration is significant and cannot be underestimated. Thus, measures should be taken to guarantee accurate and repeatable dielectric data gathering of biological tissue.

In future work, the influence of more confounders should be investigated. These results will determine which parameters have a significant influence on the measured properties and which ones can be neglected and thus should not be included in the metadata. Until an agreement on a standard operating procedure with an acknowledged metadata form, we can only encourage authors to report all metadata in their work to allow future comparisons between studies of dielectric properties of biological tissue.

\section{CONClusion}

The effect of dehydration on dielectric measurements of porcine liver as a function of time was investigated at different temperatures. Measurement data demonstrated that temperature not only has a direct effect on the measured dielectric properties but that it influences these properties in another manner as well, i.e. dehydration. During the experiment duration of 35 minutes, a significant relative change in the real as well as the imaginary part of the dielectric properties up to $9 \%$ has been established, which is significant with respect to multiple applications.

Based on food process literature, the moisture content level $X$ of samples as a function of time $t$ can be predicted with respect to temperature $T$ and air velocity $u$. In the case of high water content tissues like porcine liver in this work, the predicted trends by these equations are confirmed by the measurement data. From these equations, possible improvements are derived to stabilize the dielectric properties. By sealing the measurement container, the air flow around the sample is limited and a reduction in relative change of dielectric properties of $3.1 \%$ to $4.7 \%$ is achieved. This indicates that it is possible to extend the stability of the dielectric properties of the sample over time. In addition, this illustrates the relevance of clear documentation of sample storage between the time of excision and the measurement as well as the environmental conditions throughout the measurement. 


\section{ACKNOWLEDGMENT}

This research is supported in part by the Research Foundation Flanders (FWO) SB PhD fellowship under grant number 1S23918N (Gertjan Maenhout) and has been developed in the framework of and supported by COST Action CA17115MyWAVE. The research has also received funding from the European Research Council under the European Union's Horizon 2020 Programme/ERC Grant Agreement BioElecPro n. 637780 and the Irish Research Council Postdoctoral Fellowship Programme. The authors would like to thank the staff at the Translational Medical Device Lab in Galway for productive discussions as well as constructive feedback.

\section{REFERENCES}

[1] M. Iskander and C. Durney, "Electromagnetic techniques for medical diagnosis: A review," Proceedings of the IEEE, vol. 68, no. 1, p. 126132, 1980.

[2] D. S. Holder, Electrical impedance tomography: methods, history and applications. IoP Publishing, 2005.

[3] E. Porter et al., "An early clinical study of time-domain microwave radar for breast health monitoring," IEEE T. Bio-med. Eng., vol. 63, pp. 530539, March 2016.

[4] R. Chandra et al., "On the opportunities and challenges in microwave medical sensing and imaging," IEEE T. Bio-med. Eng., vol. 62, pp. 16671682, July 2015.

[5] J. A. Dickson and S. K. Calderwood, "Temperature range and selective sensitivity of tumors to hyperthermia: A critical review," Ann. NY Acad. Sci., vol. 335, no. 1 Thermal Chara, p. 180205, 1980.

[6] C. Simon et al., "Microwave ablation: Principles and applications," Radiographics, vol. 25, 2005.

[7] C. Gabriel et al., "The dielectric properties of biological tissues: I. literature survey," Phys. Med. Biol., vol. 41, no. 11, pp. 2231-2249, 1996.

[8] IT'IS Foundation, "Overview - database of tissue properties." https://itis.swiss/virtual-population/tissue-properties/overview/, July 2019.

[9] E. Porter et al., "Minimum information for dielectric measurements of biological tissues (MINDER): A framework for repeatable and reusable data," Int. J. RF Microw. C. E., vol. 28, no. 3, 2017.

[10] M. Lazebnik et al. , "Ultrawideband temperature-dependent dielectric properties of animal liver tissue in the microwave frequency range," Phys. Med. Biol., vol. 51, no. 7, pp. 1941-1955, 2006.

[11] A. Peyman et al., "Variation in dielectric properties due to pathological changes in human liver," Bioelectromagnetics, vol. 36, no. 8, pp. 603612, 2015.

[12] K. Foster et al., "Dielectric properties of brain tissue between 0.01 and 10 GHz," Phys. Med. Biol., vol. 24, pp. 1177-1187, Jan 1979.

[13] P. Stauffer et al., "Phantom and animal tissues for modelling the electrical properties of human liver," Int. J. Hyperthermia, vol. 19, no. 1, pp. 89-101, 2003.

[14] Z. B. Maroulis and G. D. Saravacos, Food process design. Marcel Dekker, 2003.

[15] H. Schwan and K. Foster, "Microwave dielectric properties of tissue. some comments on the rotational mobility of tissue water," Biophys. J., vol. 17, no. 2, p. 193197, 1977.

[16] R. Reinoso et al., "Tissue water content in rats measured by desiccation," J. Pharmacol. Tox. Met., vol. 38, no. 2, pp. 87-92, 1997.

[17] C. Gabriel, "Dielectric properties of biological tissue: Variation with age," Bioelectromagnetics, vol. 26, no. S7, pp. 12-18, 2005.

[18] A. Shahzad et al., "Investigation of the effect of dehydration on tissue dielectric properties in ex vivo measurements," Biomed. Phys. Eng., vol. 3, no. 4, p. 045001, 2017.

[19] D. Pollacco et al., "Characterization of the dielectric properties of biological tissues and their correlation to tissue hydration," IEEE T. Diel. El. In., vol. 25, no. 6, pp. 2191-2197, 2018.

[20] P. Meaney et al., "Open-ended coaxial dielectric probe effective penetration depth determination," IEEE T. Microw. Theory, pp. 1-9, 2016.

[21] C. Gabriel and A. Peyman, "Dielectric measurement: error analysis and assessment of uncertainty," Phys. Med. Biol., vol. 51, no. 23, pp. $6033-$ 6046, 2006.
[22] A. La Gioia et al., "Open-ended coaxial probe technique for dielectric measurement of biological tissues: Challenges and common practices," Diagnostics, vol. 8, no. 2, p. 40, 2018.

[23] A. Peyman et al., "Complex permittivity of sodium chloride solutions at microwave frequencies," Bioelectromagnetics, vol. 28, no. 4, pp. 264274,2007

[24] J. C. for Guides in Metrology, "Jcgm 100: Evaluation of measurement data - guide to the expression of uncertainty in measurement," tech. rep., JCGM, 2008

[25] S. Bell, "A beginner's guide to uncertainty of measurement," Meas. Prac. Guide, vol. 11, no. 2, 1999.

[26] M. Lazebnik et al., "A large-scale study of the ultrawideband microwave dielectric properties of normal, benign and malignant breast tissues obtained from cancer surgeries," Phys. Med. Biol., vol. 52, no. 20, p. 60936115,2007

[27] T. Sugitani et al., "Complex permittivities of breast tumor tissues obtained from cancer surgeries," Appl. Phys. Lett., vol. 104, no. 25, p. 253702, 2014.

[28] A. Fornes-Leal et al., "Dielectric characterization of healthy and malignant colon tissues in the $0.5-18 \mathrm{GHz}$ frequency band," Phys. Med. Biol. vol. 61, no. 20, p. 73347346, 2016.

[29] R. Peloso et al., "Dielectric properties of solid tumors during nonnothermia and hyperthermia," IEEE T. Bio-med. Eng., vol. BME-31, pp. 725728, Nov 1984

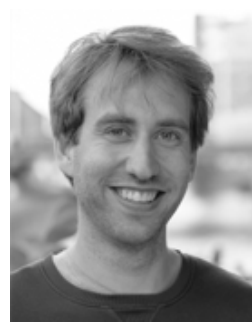

Gertjan Maenhout received the B.Sc. and M.Sc. degrees in electrical engineering from KU Leuven, Leuven, Belgium, in 2015 and 2017, respectively, where he is currently pursuing the Ph.D. degree with a focus on electromagnetic cancer detection, microwaves for life science applications, and dielectric modeling.

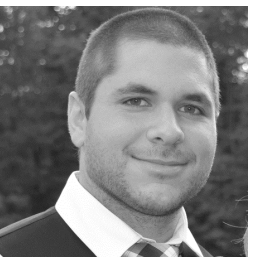

Adam Santorelli received the B. Eng. degree in electrical engineering,the M.Eng. degree in electromagnetics, and the Ph.D. degree from McGill University, Montreal,Canada, in 2010, 2012, and 2017 , respectively.

$\mathrm{He}$ is currently a Post-Doctoral Researcher with the Translational Medical Device Lab. His research interests are focused on biomedical applications with the primary goal of increasing the accessibility to technology in order to improve diagnosis, including the optimization and miniaturization of off-the-shelf components to design and fabricate custom built low-cost microwave systems. He received the 2nd Place Best Student Paper Award at the 2014 IEEE Sensors Application Symposium.

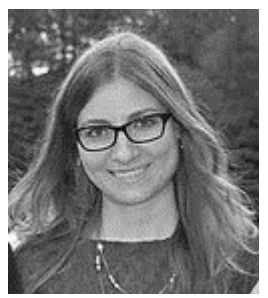

Emily Porter received her B.Eng., M.Eng., and $\mathrm{Ph} . \mathrm{D}$. degrees in electrical engineering from McGill University, Montreal, Canada, in 2009, 2010, and 2015 respectively. Dr. Porter was a recipient of the 2013 IEEE Antennas and Propagation Society Doctoral Research Award for her work on breast health monitoring using a time-domain microwave system.

Dr. Porter is currently an EU Marie-Curie Fellow with the Translational Medical Device Laboratory at the National University of Ireland Galway. Her current research interests include the measurement of dielectric properties of biological tissues and the development novel technologies for therapeutic and diagnostic applications of electromagnetic waves. 


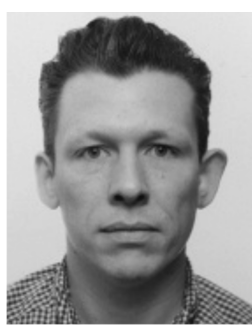

uide integration.

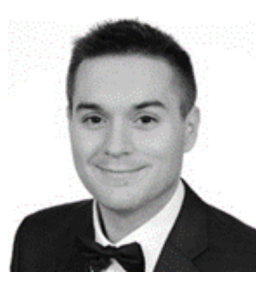

Ilja Ocket received the Ph.D. degree in Electrical Engineering from KU Leuven, Leuven, Belgium, in 2009.

He has been with imec and the KU Leuven (div. ESAT-TELEMIC) since 1999 where he has been involved in research on millimeter wave components and systems.

$\mathrm{He}$ is now active in the areas of 79 and $140 \mathrm{GHz}$ CMOS radar sensors, microwave, and millimeter wave metrology, application development for life sciences, and (sub)-millimeter wave plastic waveg-

Tomislav Markovic received the $\mathrm{Ph} . \mathrm{D}$. degree in Electrical Engineering from KU Leuven, Leuven, Belgium, in 2018. He received B.Sc. and M.Sc. degrees in electrical engineering and information technology from the University of Zagreb, Zagreb, Croatia in 2011 and 2013, respectively.

Since 2018, he is a postdoctoral research associate at KU Leuven working on various topics dealing with microwaves for biomedical and life science applications.

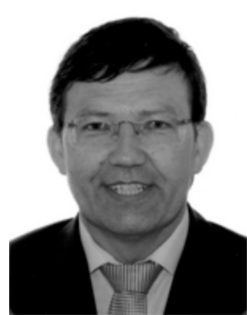

Bart Nauwelaers received the Ph.D. degree in Electrical Engineering from the KU Leuven in 1988. He also received the Masters degree in Design of Telecommunication Systems from Télécom ParisTech, Paris, France.

Since 1981, he has been with the Department of Electrical Engineering, KU Leuven, Leuven, Belgium where he has been involved in research on microwave antennas, passive components, interconnects, microwave integrated circuits and MMICs, linear and non-linear device modeling, MEMS, and wireless communications.

Dr. Nauwelaers is the former chair of IEEE AP/MTT-Benelux and past chair of URSI-Benelux. 